Conoscopic figure: a complex consequence of a not so simple phenomenon

This content has been downloaded from IOPscience. Please scroll down to see the full text. 2015 Eur. J. Phys. 36015014

(http://iopscience.iop.org/0143-0807/36/1/015014)

View the table of contents for this issue, or go to the journal homepage for more

Download details:

IP Address: 141.255.204.59

This content was downloaded on 11/12/2014 at 09:41

Please note that terms and conditions apply. 


\title{
Conoscopic figure: a complex consequence of a not so simple phenomenon
}

\author{
Maja Pečar ${ }^{1}$ and Mojca Čepič ${ }^{1,2}$ \\ ${ }^{1}$ Faculty of Education, University of Ljubljana, Slovenia \\ ${ }^{2}$ Jožef Stefan Institut, Ljubljana, Slovenia \\ E-mail: maja.pecar@pef.uni-lj.si
}

Received 6 May 2014, revised 22 July 2014

Accepted for publication 1 August 2014

Published 24 November 2014

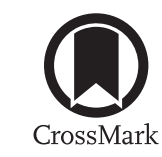

\begin{abstract}
Observation of conoscopic figures through a special microscope is widely used as an experimental method for determination of optical properties of anisotropic materials. The conoscopic figure appears when a divergent light passes through an anisotropic material sandwiched between crossed polarizers. The complex pattern of bright and dark areas appears due to different polarization states of light. The pattern gives information about optical axes and birefringence of the material and allows for deduction of microscopic structures at the origin of such properties. Due to the complexity of the figure, the method is often used following recipes. This contribution presents a series of experiments that provide students with experiences needed for construction and comprehension of optical phenomena that contribute to the complexity of the conoscopic figure.
\end{abstract}

Keywords: conoscopy, polarization states, education, simple experiments

\section{Introduction}

A semi-transparent tracing paper, one polarizer, a few layers of transparent adhesive tape or a transparency and another polarizer crossed (two polarizers called polarizer and analyser with their transmission directions set perpendicularly) with the first one are placed one onto another. If one shines a laser onto such a simple hands-on set-up from the side where the tracing paper is placed, a conoscopic figure appears on the screen as seen in figure 1. The resulting complex figure is one of the traditional methods for determination of optical properties for anisotropic materials. In addition, the artistic beauty of the figure is interesting on its own.

Before continuing, let us briefly state the reasons for such complexity. The light in a transparent anisotropic material in general splits into two beams with different phase velocities, different refractive indices and mutually perpendicular polarizations. In the experiment described above the light is divergent due to the irregularity of the tracing paper. The incident 

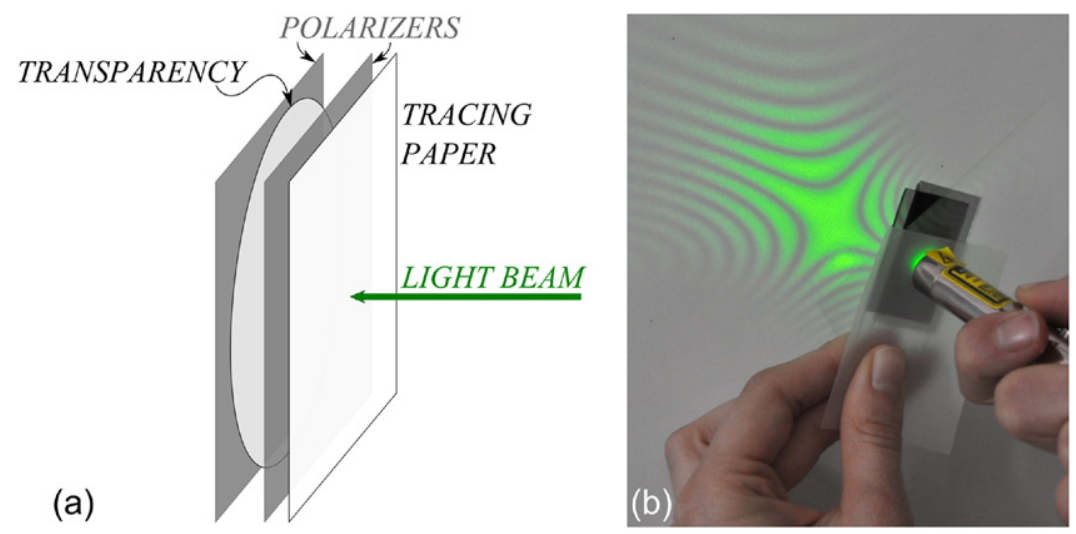

Figure 1. (a) Schematic representation of illuminating a piece of tracing paper and a transparency between crossed polarizers by a laser beam and (b) the photograph of the experiment which results in a complex figure on the screen.

light becomes polarized by the first polarizer and enters the sandwiched anisotropic material. The polarization state changes during the transmission and in general becomes elliptically polarized. The polarization state depends on the polarization of the incident light, its wavelength and its direction of incidence. The dielectric tensor of the material determines the polarization state of the transmitted light. The second polarizer partially absorbs the light, as the intensity of transmitted light through the last polarizer depends on the polarization state after transmission through the anisotropic material. The reason for the complexity of the conoscopic figure is due to the variation of polarization state in dependence of direction of the incident light.

For students to deduce anisotropic material properties from the conoscopic figure, a thorough understanding of its formation is highly beneficial. This knowledge is of special value in situations when 'cookbook recipes' do not give an unambiguous answer, for example when only a small part of a conoscopic figure can be observed.

Not many aspects of teaching and learning conoscopy as an experimental method for determination of anisotropic properties of matter have been discussed yet. Several experiments were suggested as demonstrations related to the polarization of light, mostly for linearly polarized light. Only a few of them suggest demonstrations or models of explanations for phenomena related to elliptical polarization and its construction from two orthogonal phase shifted linearly polarized light beams [1-3]. More studies were found related to the determination and presentation of different states of elliptical polarization [4-6].

Conoscopic figures were discussed in [7-14]. The authors considered simple hands-on experiments that allowed for an observation of conoscopic figures of transparencies [7-9] and simple arrangements for observing conoscopic figures of crystals [10-14]. More details were given for a theoretical analysis of conoscopic figures $[9,13,15]$ and propagation of electromagnetic waves through other anisotropic media [16, 17]. All references considering conoscopic pictures were focused on understanding its meaning. Other papers were focused on specific properties of simple anisotropic material and their measurements [18-21]; however they were not related to conoscopic figure formation. The main goal of this paper however is to present a set of experiments, which combined in a proper sequence provide experience with optics of anisotropic materials to students and help them understand and comprehend the formation of the conoscopic figure. 
The paper is organized as follows: in section 2 we briefly resume the optics of anisotropic materials and description and presentation of polarization states. We derive expressions that link properties of the polarized incident light, the anisotropic material and the intensity of the transmitted light through the second polarizer. We also describe the conoscopic figure and introduce the nomenclature that is used in the continuation. In section 3 we present the sequence of experiments that explains separately several phenomena merging in the conoscopic figure. We apply the results to discussion of additional experiments allowing for a deeper insight into the method and the data the conoscopy can provide. Section 4 concludes the report and discusses few suggestions that could be used for laboratory work. As the paper discusses several different phenomena, experiments are distinguished by the main goal of the experiment given in italics and with a bold experimental number.

\section{Theoretical background}

In this section we shortly review fundamentals of phenomena essential for understanding the conoscopic figure. The section does not report any new physics but should help the reader with a general physics background to remember or to become acquainted with the nomenclature used later.

\subsection{Properties of anisotropic materials}

Electrical properties of anisotropic materials are described by a dimensionless symmetric dielectric tensor $\underline{\varepsilon}$ that connects an electric displacement vector $\vec{D}$ and the electric field $\vec{E}$.

$$
\underline{\varepsilon}=\left(\begin{array}{lll}
\varepsilon_{x x} & \varepsilon_{x y} & \varepsilon_{x z} \\
\varepsilon_{y x} & \varepsilon_{y y} & \varepsilon_{y z} \\
\varepsilon_{z x} & \varepsilon_{z y} & \varepsilon_{z z}
\end{array}\right), \quad \vec{D}=\underline{\varepsilon} \vec{E} .
$$

Three mutually perpendicular directions exist for which a direction of an electric displacement vector is parallel to the applied electric field. These three directions correspond to the eigenvectors of the three eigenvalues $\left(\varepsilon_{1}, \varepsilon_{2}, \varepsilon_{3}\right)$ of the dielectric tensor, and are called also principal axes. If the axes of the coordinate system in which an analysis is considered coincide with eigenvectors, one says that the phenomenon is studied in the eigensystem and the dielectric tensor adopts a very simple form.

$$
\underline{\varepsilon}=\left(\begin{array}{ccc}
\varepsilon_{1} & 0 & 0 \\
0 & \varepsilon_{2} & 0 \\
0 & 0 & \varepsilon_{3}
\end{array}\right)=\left(\begin{array}{ccc}
n_{1}^{2} & 0 & 0 \\
0 & n_{2}^{2} & 0 \\
0 & 0 & n_{3}^{2}
\end{array}\right) .
$$

Here $n_{1}, n_{2}$ and $n_{3}$ are the corresponding refractive indices. The solution of wave equations for propagation of light through an anisotropic material shows that in any direction light propagates with two different beams having two different phase velocities and mutually perpendicular polarizations. In general, the two velocities, the phase (wave-normal) velocity and the ray (energy) velocity are different in magnitude and directions [22]. As our discussion is limited to the phase velocity that defines changes of phase during the propagation and the refraction of light in an anisotropic material, we do not discuss these subtleties in further details. The phase velocity we simply call the speed of light.

In this paragraph we will illustrate the phenomenon in a specific geometry. Let the principal axes correspond to $x_{0}, y_{0}$ and $z_{0}$ axes of the coordinate system in which we consider this example. The dielectric tensor has the same form as in equation (2). The light propagates 
along the $z_{0}$ axis. The polarizations of the two beams are parallel to the other two principal axes, $x_{0}$ and $y_{0}$ and their phase velocities are $c_{0} / n_{1}$ and $c_{0} / n_{2}$, respectively. For any other direction the situation is the same. The light propagates in two parallel beams with two phase velocities and two mutually perpendicular polarizations. The only difference is that both phase velocities and corresponding polarizations are not so straightforward as in the presented example. They have to be calculated taking the geometry of the problem into consideration. Finally, the ratio between the speed of light in the vacuum and the speed of light in a transparent anisotropic material for a specific direction and a corresponding polarization defines the index of refraction for the light beam. Therefore the incidence light from an isotropic medium splits into two perpendicularly polarized beams that propagate with different speeds and in different directions.

In every transparent anisotropic material directions exist in which the light propagates as in an optically isotropic material, that is, the speed does not depend on the polarization of the beam. These directions are called optical axes. In materials where two of eigenvalues for $\underline{\varepsilon}$ are equal, only one such direction exists and such materials are called uniaxial. If all three values of $\underline{\varepsilon}$ differ, two such directions of light propagation exist and the material is called biaxial.

Another point with respect to nomenclature is worth mentioning. The numerical difference between the minimum and the maximum values of the refractive indices is called the birefringence of the material. Sometimes researchers are not very strict when they use the term birefringence. The expression 'birefringence' is often used for the difference of the two refractive indices for the two polarizations in the considered direction of light propagation. If the term birefringence is used in such a way, it is direction dependent. In this paper we use the expression birefringence in its original meaning as the difference between the largest and the smallest refractive index of the material $[22,23]$.

\subsection{Polarization states}

Students get acquainted with polarization as an essential property of electromagnetic and other transversal waves. Equations are always written for waves with well determined directions of oscillating electric field in time. The fact that light is usually not polarized is mentioned when polarization is introduced. Textbooks and lecturers mostly consider other types of polarization, such as the circular and the elliptical for very specific geometries. A typical example would be where the linear polarizer is placed under $45^{\circ}$ with respect to vibration directions and a derivation of the polarization state for the transmitted light leads to specific examples of $\lambda$-quarter and $\lambda$-half plates. A smart student can easily apply the approach to more general examples but without being forced to practice not so straightforward examples, both theoretically and experimentally, students usually forget that the polarization direction of the incident light also significantly affects the polarization state of transmitted light. The conoscopic figure is a result of such a variation. As light has different incident directions with respect to the principal axes of the material, a large variety of situations combines in the final figure. As polarization state of the transmitted light is very important for understanding of a conoscopic figure, we review in this subsection the theoretical and graphical presentation of polarization states. The analysis is made for perpendicularly incident light although the conclusions are valid for a superposition of any two phase shifted mutually perpendicularly polarized beams and therefore for the propagation of light in any direction.

The most obvious description of the polarization state is if the coordinate system, in which the analysis is done, corresponds to two polarization directions called also directions of vibration or vibration directions, say $x$ and $y$, that determine the two phase velocities and the 


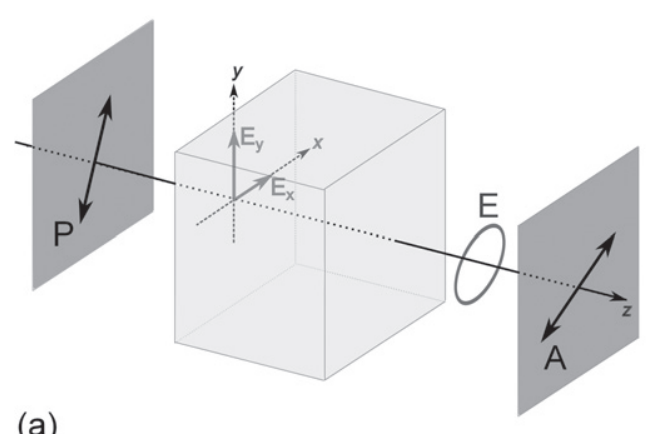

(a)

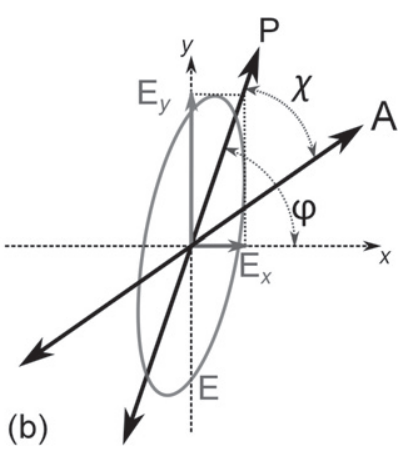

(b)

Figure 2. (a) Schematic representation of an experiment for studying effects of anisotropic material (light grey) on polarized light. Double sided arrows mark transmission directions of polarizer $(\mathrm{P})$ and analyser (A). The light in the anisotropic material splits into two beams polarized along $x$ and $y$ directions. Characteristic ellipse E illustrates the polarization state of light after passing the polarizer and the anisotropic material. (b) Polarization state of transmitted light and a geometry corresponding to the situation on the left.

corresponding wave vectors along the $z$ th axis. This coordinate system does not necessary correspond to the principal axes of the material and depends on the direction of propagation of light in an anisotropic material.

After propagating through the anisotropic material, the two electromagnetic waves have the same wavelength but they in general differ in phase and in the amplitude:

$\vec{E}(\vec{r}, t)=\left\{E_{0} \cos \varphi \cos \left(\omega t-k z-\delta_{x}\right), E_{0} \sin \varphi \cos \left(\omega t-k z-\delta_{y}\right)\right\}$.

Here $\varphi$ is the angle between a direction of polarization of the incident beam and the $x$ axis as seen in figure 2(b). The electromagnetic wave of angular frequency $\omega$ propagates in the vacuum with a wave vector $k$. As the two polarizations in the material have different phase velocities and thus the material has different optical lengths for the two polarizations, the two polarizations acquire different phase shifts when leaving the material, $\delta_{\mathrm{x}}$ and $\delta_{\mathrm{y}}$, respectively. The presentation of the electric field is further simplified by renormalization of time that sets the whole phase of the component $E_{x}$ at the coordinate $z$, where the light passes the interface from anisotropic material to air to zero:

$$
\vec{E}(\vec{r}, t)=\left\{E_{0} \cos \varphi \cos (\omega t-k z), E_{0} \sin \varphi \cos (\omega t-k z-\delta)\right\} .
$$

In equation (4), $\delta=\delta_{y}-\delta_{x}$ is called the phase difference.

The polarization state of light is usually visualized by a curve in the shape of an ellipse called also a characteristic ellipse that is traced by a tip of the electric field vector during one period at a constant position $z$. Figure 3 shows polarization states for various orientations of the incident polarizations $\varphi$ and for various phases $\delta$.

Let us discuss polarization states presented in figure 3. One can easily see that for general angles $\varphi$ and for general phase differences $\delta$ the characteristic curves drawn by the end of the electric field vector is an ellipse. The light is then called elliptically polarized light. For specific cases the ellipse degenerates to a line (if $\varphi=0$ or $\pi / 2$ or $\delta=k \pi$ where $k=0, \pm 1, \pm 2, \ldots$ ), which means that electric field oscillates in one plane and the light is called linearly polarized. Finally, for a very specific case, when amplitudes of oscillations in both perpendicular directions are the same $(\varphi=\pi / 4)$ and the phase difference is exactly 


\begin{tabular}{|c|c|c|c|c|c|}
\hline & $\delta=0$ & $\delta=\mp \pi / 4$ & $\delta=\mp \pi / 2$ & $\delta=\mp 3 \pi / 4$ & $\delta=\mp \pi$ \\
\hline$\varphi=\pi / 8$ & & E & $E$ & & Z \\
\hline$\varphi=\pi / 4$ & & & & & \\
\hline$\varphi=3 \pi / 8$ & $\mathrm{E} /$ & $y_{E}^{y}$ & & & \\
\hline
\end{tabular}

Figure 3. Different polarization states. Angle $\varphi$ is associated to the magnitudes of electric field along $x$ and $y$ directions. The $\mp$ sign in the phase difference $\delta$ distinguishes between the left and the right handedness of the elliptically or circularly polarized light.

$\delta= \pm \pi / 2$, the ellipse has equal diameters, i.e., it is a circle and the light is called the circularly polarized light.

In a typical experimental setup in students' laboratories the polarizer is set at $45^{\circ}$ with respect to one of the vibration directions of the anisotropic material and students may get a false impression. By increasing the thickness of the anisotropic material, the phase difference $\delta$ increases. The elliptical polarization changes through circular polarization (the $\lambda$-quarter plate) to linearly polarized light that is rotated for $90^{\circ}$ (the $\lambda$-half plate) with respect to the incident polarization. The long axis of the characteristic ellipse changes to the short axis, however its direction remains parallel to the direction of the incident polarization. This is a particular case. For any other direction of incident polarization the length of the long axis of the characteristic ellipse changes but it also rotates upon increasing the phase difference $\delta$.

The initially polarized light that has propagated through an anisotropic medium generally changes its state of the polarization and becomes elliptically polarized. The polarization state is described by two parameters (figure 3): the angle $\varphi$ that gives the orientation of the polarizer with respect to the vibration directions and the phase difference $\delta$ that occurs due to different optical lengths of the two beams with different phase velocities:

$$
\delta=\delta_{y}-\delta_{x}=2 \pi \frac{d}{\left(\lambda_{0} / n^{\prime}\right)}-2 \pi \frac{d}{\left(\lambda_{0} / n^{\prime \prime}\right)}=2 \pi\left(n^{\prime}-n^{\prime \prime}\right) \frac{d}{\lambda_{0}} .
$$

Here $\lambda_{0}$ is the wavelength of light in vacuum, $d$ is the material thickness (for perpendicular incident light), and $n^{\prime}, n^{\prime \prime}$ are refractive indices for two specific polarizations of the two beams in which the light splits.

Although the analysis above was made for a special geometry it is valid generally. Let us repeat, for any direction of light propagation in the anisotropic medium, two mutually 

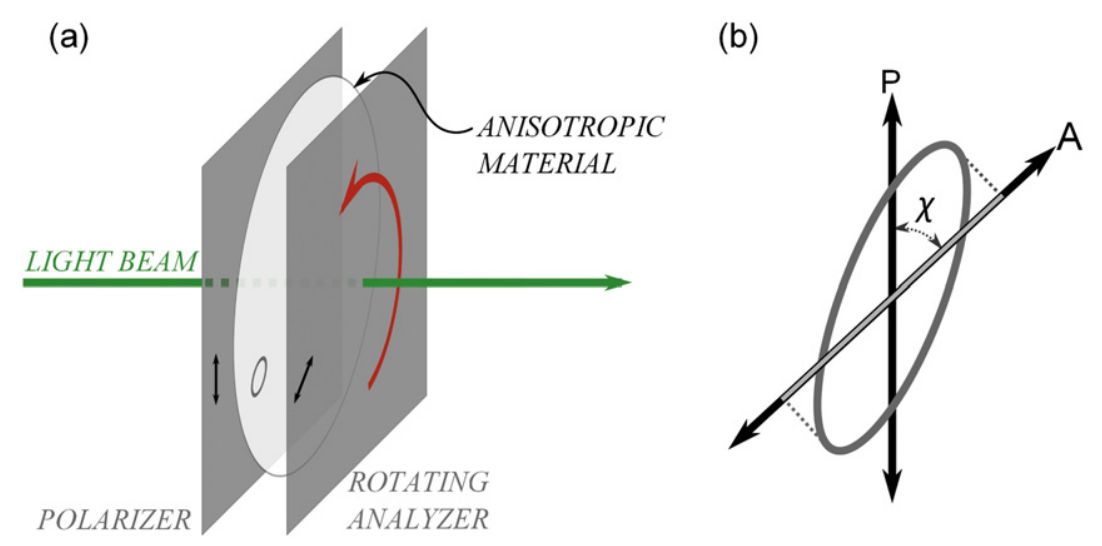

Figure 4. (a) Schematic representation of the experimental set-up for the measurement of the intensity of the transmitted light while rotating the analyser. (b) Intensity of light is proportional to the projection of the characteristic ellipses on the transmission direction of the analyser as marked by dashed lines and brighter line on the analyser.

perpendicular directions that coincide to polarizations of the two beams can be associated to $x$ and $y$ axes. For these two directions two in general different phase velocities are associated and define two different refractive indices, say $n^{\prime}, n^{\prime \prime}$ [22].

A very nice and instructive visualization of the polarization state changes during the propagation of light through an anisotropic material is the animation called EMANIM available at [24].

To summarize, any linearly polarized light that passes an anisotropic material generally changes its state of polarization. The state of polarization after transmission depends on the initial polarization and the wavelength of the incident light; on the properties of the anisotropic sample, through which the light propagates, and its thickness; finally it depends on the direction of light propagation in the material and with it associated two refractive indices.

\subsection{Determination of polarization states}

There are several means to analyse the state of polarization. One may use linear polarizers, circular polarizers, retardation plates and their combinations. Conoscopic figure is composed from light beams with different directions transmitted through an anisotropic material between the crossed polarizers. The second polarizer, called the analyser, is also used to detect the polarization state of a single light beam passing through the material. Therefore such analyser can be helpful for better understanding of the transmitted intensity of light at different places in conoscopic figure.

The polarization state is detected by rotation of the analyser accompanied by the measurement of the intensity of transmitted light as shown in figure 4. Here it is not necessary that the incident light is perpendicular to the anisotropic material as long as the material is rather thin.

The measurements of intensity of transmitted light with respect to the orientation of the analyser are presented in two different ways. Figure 5(a) presents a dependence of the intensity of the transmitted light on the direction of the analyser's transmitting direction given by the angle $\chi$. Both maxima and minima give the orientation of the major and minor semiaxes of the characteristic ellipse describing the state of polarization. The same is true for a presentation given in figure 5(b), but the graph is polar and the properties of the ellipse are 
(a)

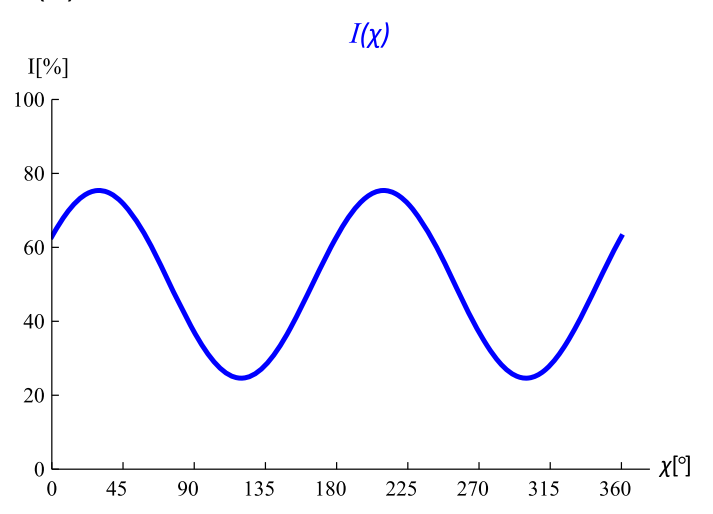

(b) $\quad I(\chi)$

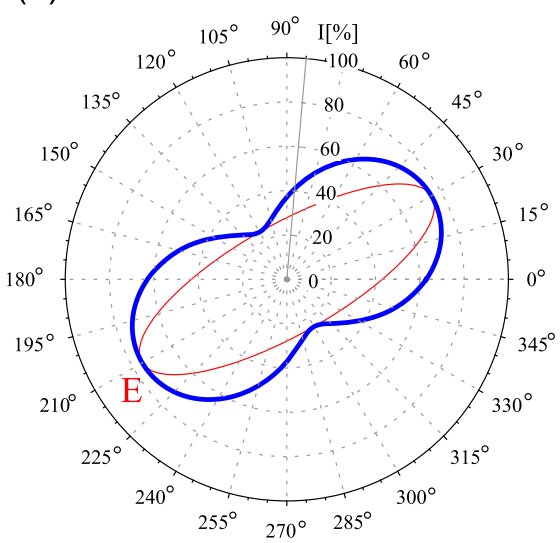

Figure 5. Two different graphical presentations of an elliptical state with the phase difference $\delta=90^{\circ}$ and the orientation of the vibration direction due to the incidence linear polarization $\varphi=120^{\circ}$ measured as transmitted intensity (in percents) while rotating the analyser $(\chi)$. (a) Dependence of the intensity in the transmitted signal (vertical axis) on the orientation of the analyser (horizontal axis). (b) The same dependence given as a polar graph, where the blue curve is the transmitted intensity in dependence of the orientation of the analyser $\chi$, the red curve is the average electric field or better the characteristic ellipse of the polarization state, which is not directly proportional to the the intensity, because the intensity of the transmitted light is determined by the average of the square of electric field.

even easier to recognize as marked in the figure with a red inner curve. The graphical presentation of the electric field amplitude corresponds to the characteristic ellipse, while the presentation of the intensity of the transmitted light is proportional to its square.

The study of the polarization state of light presented above allows for determination of optical properties of anisotropic material. Let us study the intensity of light in a coordinate system as shown in figure 2(b). The intensity of light $I(\varphi, \chi, t)$ after passing the analyser is obtained from the superposition of two (monochromatic) waves with phase difference $\delta$ after transmission through the anisotropic material and it is proportional to $E^{2}(\varphi, \chi, t)$. The time average of the electric field and therefore the intensity is given with the equation

$$
\begin{aligned}
I(\varphi, \chi)= & E^{2} \cos ^{2} \varphi \cos ^{2}(\varphi-\chi)+E^{2} \sin ^{2} \varphi \sin ^{2}(\varphi-\chi) \\
& +2 E^{2} \sin \varphi \cos \varphi \sin (\varphi-\chi) \cos (\varphi-\chi) \cos \delta
\end{aligned}
$$

or simplified

$$
I=I_{x}+I_{y}+2 \sqrt{I_{x} I_{y}} \cos \delta,
$$

where the amplitude $E$ is proportional to the amplitude of the $E_{0}$ presented in the equations (3) and (4). Here $I_{x}=E^{2} \cos ^{2} \varphi \cos ^{2}(\varphi-\chi)$ and $I_{y}=E^{2} \sin ^{2} \varphi \sin ^{2}(\varphi-\chi)$ are the intensities of the two perpendicularly polarized waves after passing the analyser. Using the identity $\cos \delta=1-2 \sin ^{2}(\delta / 2)$, we get

$$
I=E^{2}\left(\cos ^{2} \chi-\sin 2 \varphi \sin 2(\varphi-\chi) \sin ^{2} \frac{\delta}{2}\right) \text {. }
$$

The conoscopic figure studies the transition of light through crossed polarizers, therefore the sample is studied for one very specific situation, where $\chi=\pi / 2$. The equation (7) for this 
condition is much simpler and is reduced to

$$
I_{\perp}=E^{2} \sin ^{2} 2 \varphi \sin ^{2} \frac{\delta}{2}
$$

The latter case allows for determination of vibration directions in an anisotropic material. If one of the polarizers, either analyser or polarizer is parallel to one of the vibration directions, the light is completely absorbed. The two directions are straightforwardly found if the material is anisotropic only. If a material is optically active as well, the two directions are not so straightforward. In general, when studying a sample, vibration directions are not known, therefore also details about the polarization state do not satisfy to estimate the phase difference $\delta$ and the difference in refractive indices. As the analysis above always starts from an electric field with components along the two known principal axes students often get a false impression that by knowing the shape, i.e. both semiaxes of the ellipse and the orientation of the ellipse, all information can be extracted. The fact that the same shape of the ellipses can be obtained for different phase differences is often overlooked [22].

\subsection{The conoscopic figure}

In previous subsections we used simplified examples where the incident light was always perpendicular to the sample surface. Such observations are called orthoscopic.

The conoscopic figure is a different type of observation. The incident light is oblique with different incident angles and different planes of refraction. The observation is rather local as a smaller area of the sample is studied, but the complex picture (figure 1) gives information about light that is transmitted in a large variety of directions through the small but specific area of the sample. Therefore a direction dependence of optical properties is measured by a single measurement.

The professional conoscopy controls the direction of the incident light by focusing the light beam onto the surface of the sample. The oblique light refracts into two beams that separate in space after transmission. After leaving the material both beams are again parallel and they superpose (figure 6). An additional lens allows for a formation of a (sharp) conoscopic picture on the screen $[25,26]$.

Another method, less accurate but still appropriate for use in the laboratory or for demonstrations for educational purposes, is the following: instead of focusing the light on the sample, the laser beam passes a piece of normal tracing paper that is placed right before the polarizer and the sample (the configuration of the set-up in the figure 1(a)). In this way the light transmitted through the tracing paper has all directions (the incident light on the sample is represented in figure 6). The difference with the former method is that light is incident on a wider area of the sample and the setup is not so spatially accurate. In addition, the mutually perpendicularly polarized waves that are superposed actually correspond to different areas of the sample. Still, if the sample is thin (the thickness of the sample is marked with $h$ in figure 6), the spatial difference is not very large and the accuracy of the setup is more than satisfactory for educational purposes.

Let us introduce some vocabulary that will be needed.

The pattern that appears on the screen is formed from curves of equal intensity of the monochromatic light. Those curves are called isochromes. The name becomes evident if a conoscopic figure is obtained by a white light, because they are curves of equal colour.

Another series of lines is important for understanding of the conoscopic figure. The isogyres connect positions in the conoscopic figure that have constant angle between the 


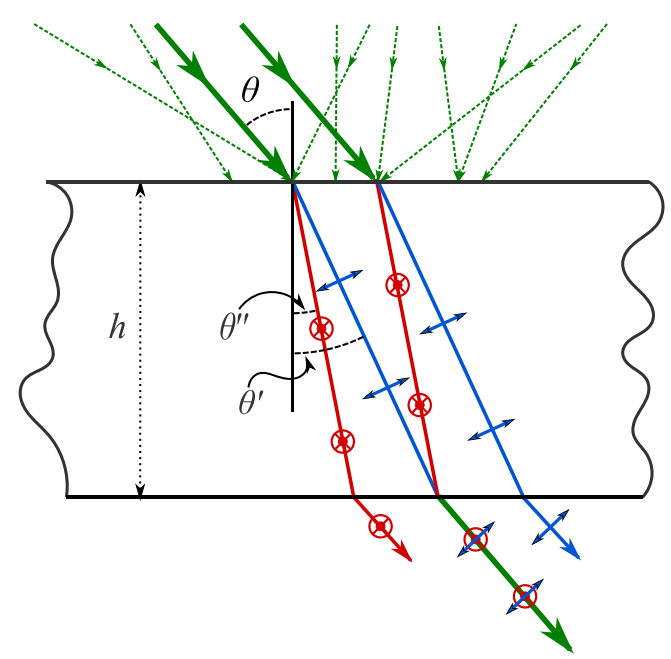

Figure 6. Schematic representation of the superposition of the two perpendicularly linearly polarized light beams in the sample while using a less accurate method illuminating an area of the material through a diffuser (tracing paper or similar, not shown in the representation). The concept of the superposition of the two perpendicularly polarized waves (red and blue schematic lines) is the same in both cases (the professional and less professional one).

polarization direction of the polarizer and one of the two vibration directions in the material (angle $\varphi$ in figure 2(b)).

As mentioned before, there are two (or one only) directions in the crystal in which both polarizations have the same speed and the phase difference does not occur, called optical axes. As the polarizer and the analyser are crossed, in those directions there is no phase difference and the black spots appear surrounded by circles of monochromes. These spots are called melatops. Uniaxial materials have one melatope only and biaxial materials have two.

After this introduction of the optics of anisotropic materials, introduction of typical expressions that are used in this field and a description of the conoscopic figure, we are prepared to dissect the conoscopic figure into its components by a set of experiments. Our aim is to provide students with experimental experiences on the influences mentioned above and help them to construct understanding of the complex conoscopic picture by applying the experience to the new more complex situation, where various phenomena are merged resulting in one complex and beautiful figure $[25,26]$.

\section{Getting experiences through experiments}

The first students' encounter with a conoscopic figure (figure 1) is usually accompanied by a wow effect due to its beauty and complexity but also because of simplicity of the experiment. However, for understanding the reasons for the complexity of the figure, more effort is required. To fully comprehend the conoscopic figure and how the anisotropic properties of the material are extracted from them, one should understand the following:

(i) Why does the intensity of the transmitted light change from one position on the figure to another? 
(ii) What influence does the change of the polarization state have and how can it influence the transmission of light?

(iii) Why does the polarization state change with the incident angle but also by changing the plane of refraction?

and in addition

(iv) Which data about the material are needed and how can they be extracted from the conoscopic figure?

(v) What happens if instead of the monochromatic light, white light is used?

In the following sections we present a set of experiments, which allow students to construct concepts that later intertwine in the complex conoscopic figure, separately. With the help of experiences gained by experiments students should be able to give explanations to questions (i)-(iii), while the last two questions can be used as project work using the new knowledge in novel circumstances.

\subsection{Initial polarization and the outgoing elliptical state}

The conoscopic figure is a visualization of angular dependence of the transmitted light intensity. The intensity can vary due to two reasons straightforwardly seen in equation (8): the initial polarization given by $\varphi$ and the phase difference $\delta$. As already mentioned, because the setups are usually met with a polarization in $45^{\circ}$ with respect to vibration directions, students get a strong impression that the polarization state is defined solely by the sample. With the following experiment students become aware that with an anisotropic sample several if not even any state of elliptical polarizations can be achieved. This experience is related to isogyres in a conoscopic figure and the recognition of directions where one of the polarizers is perpendicular to the vibration direction of a beam.

If the light is elliptically polarized, the intensity of transmitted light through an analyser is always larger than zero and it depends on the orientation of the analyser. Two orientations of analyser where the intensity of a transmitted light have the maximum and the minimum indicate orientations of the major and minor semiaxes of the characteristic ellipse (figure 4(b)). With azimuthal changes (angle $\psi$ in figure 8 ) of linearly polarized incident light to the conoscope setup, the components of electric field along vibration directions change as well. Therefore the elliptical state is influenced by the relative orientation of a polarizer with respect to polarizations of waves. To make students aware that the effect of the polarization direction is important, we suggest the experiment 1: different elliptical states obtained by a single material sample.

A single sheet of an anisotropic material is used. The sample serving the purpose the best is a simple $\lambda / 4$ (lambda-quarter) plate available for educational purposes. The laser pointer that has the closest possible wavelength to the characteristic wavelength of the lambda-quarter plate is used. If a pointer with a characteristic wavelength is not available, the fundamental message that a large variety of elliptical polarizations can be obtained by a single sample, can still be shown. The experimental set-up is presented in figure 4(a). The $\lambda / 4$ plate is placed between the two polarizers. Principal axes of the lambda-quarter plate are usually marked, but if one uses a plastic foil or a piece of a transparency instead, axes are found by rotating the sample between the crossed polarizers until the sandwich becomes completely dark (experiment demonstrated later in figures 8 and 9). The polarizer is oriented at various angles with respect to the vibration directions. The analyser is rotated and the intensity of the transmitted light is measured by a detector. Three examples of measurements are shown in figure 7. Although the sample is the same and therefore the phase difference is the same as it 
(a)

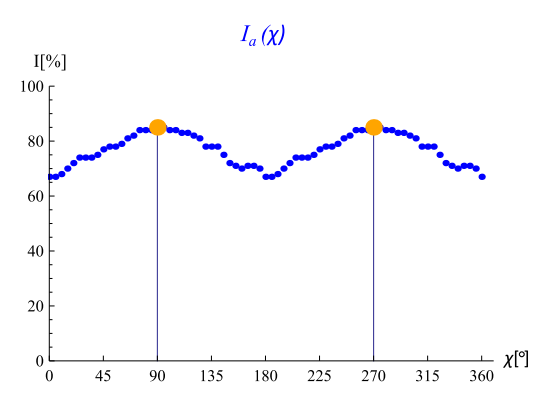

(b)

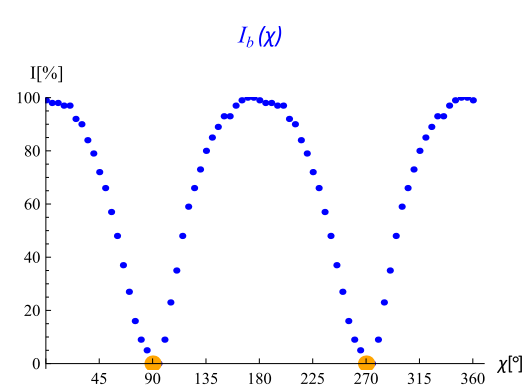

(c)

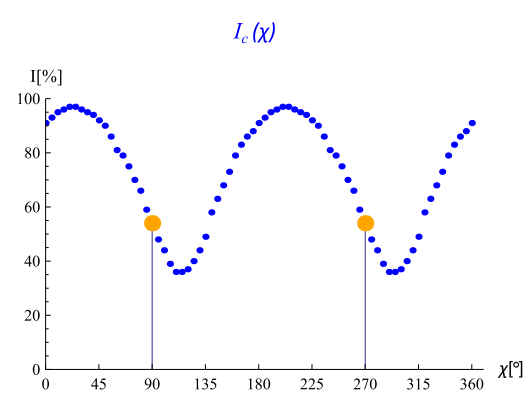

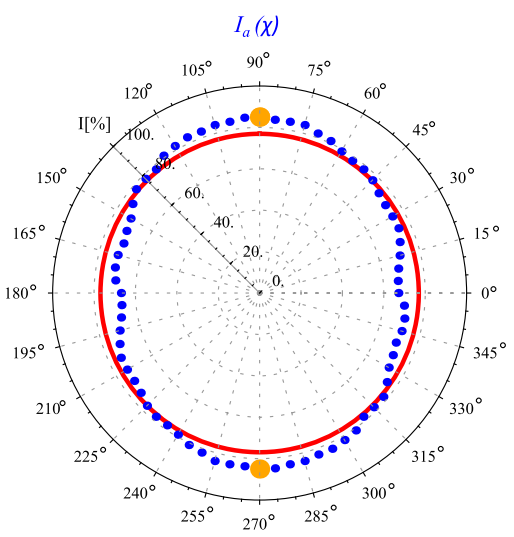

$I_{b}(\chi)$

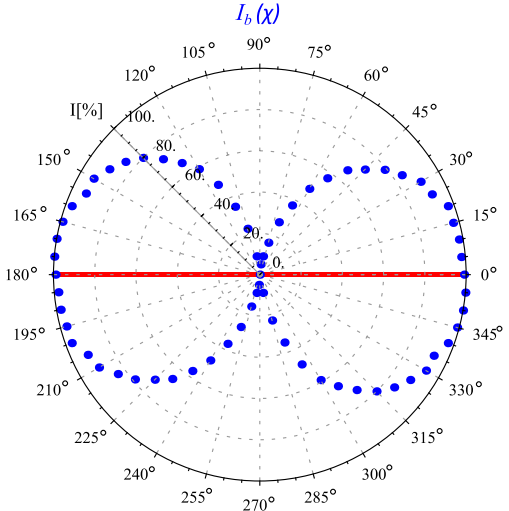

$I_{c}(\chi)$

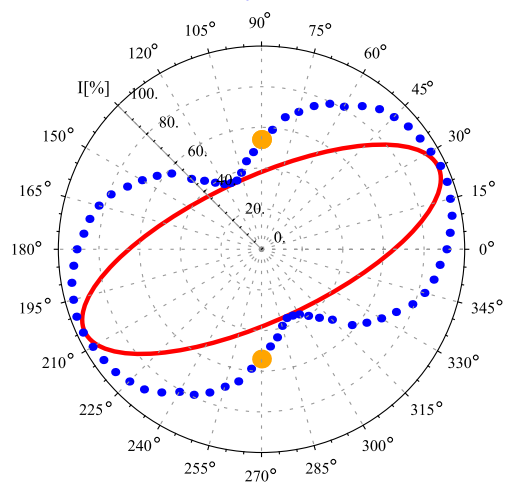

Figure 7. The intensity of transmitted light through an anisotropic material (quarterwave plate for $\lambda=560 \mathrm{~nm}$ ) in dependence on the orientation of analyser $(\chi)$ and the schematic representation of the polarization state as the characteristic curve (red curve). (a) The incident light is polarized at $45^{\circ}$ with respect to the axes of the lambda quarter plate. The transmitted light is almost circularly polarized as the green laser with $\lambda=532 \mathrm{~nm}$ was used. (b) The polarization along one of the principal directions remains linearly polarized and (c) a general polarization direction of the incident light results in elliptically polarized transmitted wave. In all figures the measurement for the crossed polarizers that correspond to the conoscopy setup are marked by an orange dot. 
depends on material and its thickness for the orthogonal monochromatic light, states of elliptical polarization are very different-from almost circular to linear-due to the orientation of the incident polarization plane.

This set of experiments shows that the vibration direction influences the final polarization state significantly (columns in figure 3 ) even if the phase difference between the two waves is constant. In the conoscopic figure only one situation (the crossed polarizers) marked in figure 7 with orange dots is present. If crossed polarizers coincide with vibration direction, only one wave is present and the light is not transmitted through the system (figure 7(b)). In the conoscopic figure this situation corresponds to two isogyres that are dark. The isogyre line corresponds to different elliptical states for which the phase difference $\delta$ varies along the isogyre but the vibration direction with respect to the incident polarization in the wave, i.e. $\varphi$ is constant. Elliptical states for such a condition are presented in lines of figure 3.

In a conoscopic figure the intensity of illumination changes from one point to another. This is because the relative orientation of the incident polarization with respect to the vibration directions change upon the incident angle and its azimuth but also due to the phase difference changes for each direction of the incident light. We shall focus on these influences in experiments in the continuation.

\subsection{Directions of vibration of the two waves}

The orientations of the two mutually perpendicular vibration directions of the two waves change with direction of propagation in general. In order to define the direction of light propagation let us first define a coordinate system to define the azimuthal incidence of light (angle $\psi$ ). For perpendicularly incident light $(\theta=0)$ two orientations of a sample between crossed polarizers, perpendicular to each other, exist for which the light is not transmitted and they will be represented as $u$ and $v$ (see figure 8).

In more detail, the set-up for the experiment consists of a laser beam, a light intensity detector and an anisotropic material between two crossed polarizers. The laser beam is fixed in the normal direction with respect to polarizers and the sample. The sample is rotated between crossed polarizers and the intensity of transmitted light is measured. Orientations of polarizer and analyser, where the intensity of the transmitted light is negligible are chosen as $u$ and $v$ axes, and the normal corresponds to the $n$th axis of the system that is used for the analysis in the continuation. The experiment used for determination of the coordinate system is similar to the one used in $[18,19]$. The direction of the light propagation is now defined by an angle $\theta$ between the propagation direction and the normal to the sample that is the $n$th axis (figure $8(\mathrm{c})$ ). The azimuth of the propagation direction $\psi$ is an angle between the projection of the propagation direction and the $u$-axis. Figure 8 (c) illustrates the meaning of the angles for an example of the oblique incident light.

For perpendicularly incident light the vibration direction of the two waves in the material corresponds to the $x$ and $y$ axes. For an oblique incidence on an anisotropic material the propagation direction changes as well as vibration directions do not lie in $x z$ or $y z$ planes any more. As most of the laboratory experiments with anisotropic materials are performed with perpendicularly incident light, this phenomenon remains often overlooked. Experiment 2, the dependence of vibration direction on direction of light propagation, provides straightforward evidence for the rotation of vibration directions in an anisotropic material for an oblique incidence of light.

Biaxial material like a piece of a transparency is taken. The experiment is set up on the optical bench in order to allow for accurate rotations of the polarizer, the anisotropic sample and the analyser, figure 9. The transparency (marked as $\mathrm{T}$ in figure 9 in the direction of its 

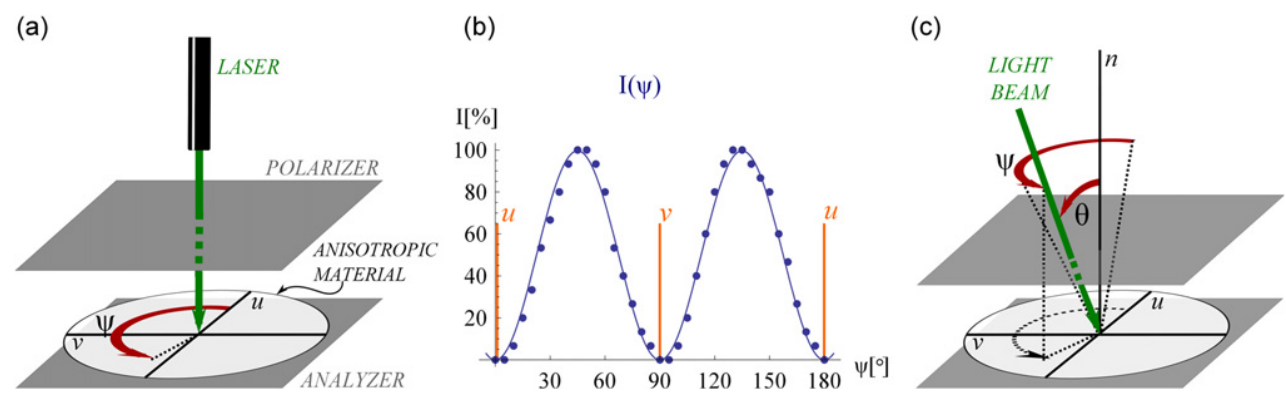

Figure 8. (a) A schematic presentation of the experimental set-up for the determination of the coordinate system. (b) The relative intensity of transmitted light of a green laser through a layer of transparent adhesive tape between two crossed polarizers in dependence of the rotation angle $(\psi)$ of the adhesive tape and its trend line proportioned to $\sin ^{2} 2 \psi$ because in this case the angle $\psi$ corresponds to $\varphi$ and the mentioned dependence can be seen from the equation (7). A photodiode and a voltmeter were used as the detector and a green laser as light emitter. (c) The schematic presentation of the orientation of the incidence light with the angles $\psi$ and $\theta$.

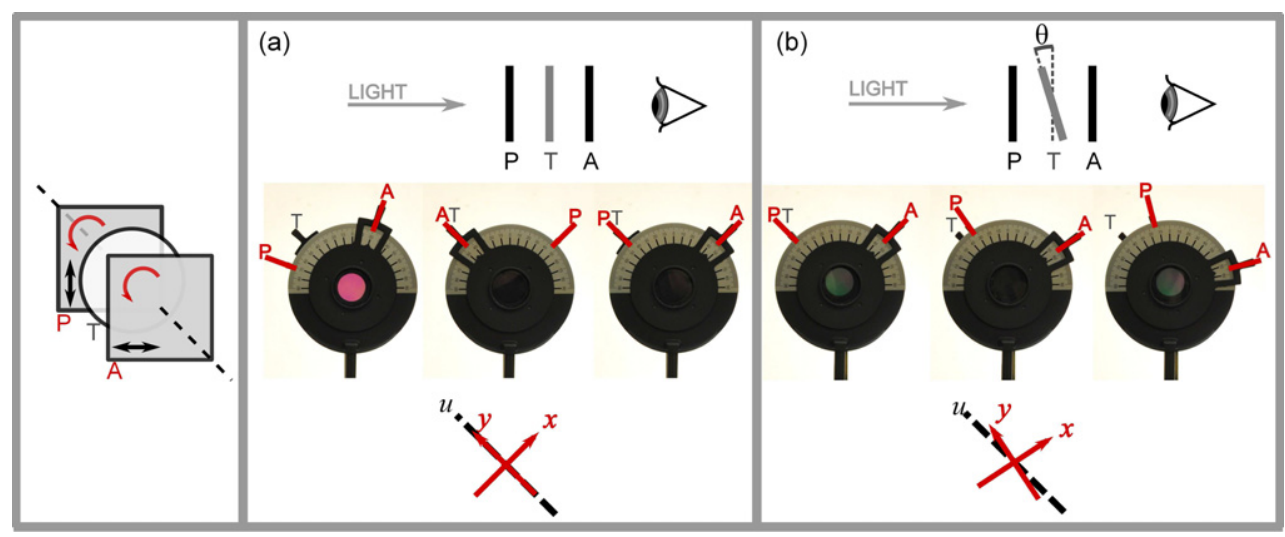

Figure 9. Photos (in the middle) and schematic representations (above) of the experiment of searching the directions of the vibration directions, where a transparency (T, with its orientation $u$ ) in between a polarizer (P) and an analyser (A) is used. (a) Some light passes through the transparency (anisotropic material) between two crossed polarizers (A and $\mathrm{P}$ ). The vibration directions (schematic representation below the photos) are found with the rotation of the still crossed polarizer and analyser (schematic representation left) where no light passes through. (b) The transparency is tilted so the light is entering it at the angle $\theta=15^{\circ}$ and $\psi=45^{\circ}$ and the light is again passing through. As in the (a) case the two vibration directions are found and are differently oriented as in (a).

axis $u$ ) is positioned between the crossed polarizers (their transmission directions are marked by $\mathrm{A}$ and $\mathrm{P}$ in figure 9). The polarizer and the analyser are rotated in order to find the orientations where the transmission is negligible ( $x$ and $y$ axes), which for perpendicularly incident light correspond to the $u$ and $v$ axes (figure 9(a)). Next, the sample is slightly rotated around the vertical axis-the holder with a transparency is rotated (figure 9(b)). In the new set-up the light is not absorbed in general. The mutually perpendicular polarizer and the 
(a)

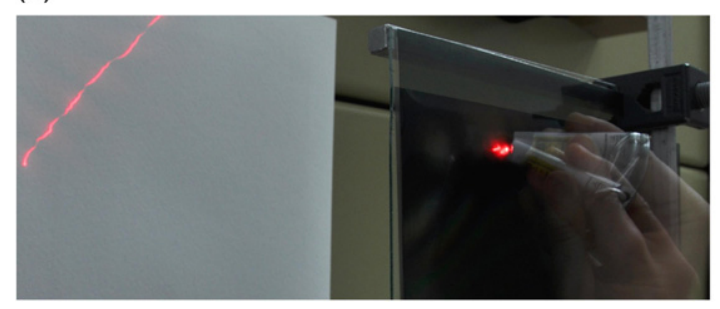

(b)

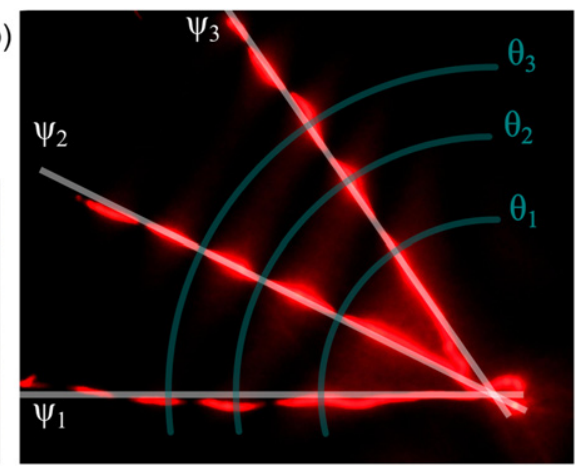

Figure 10. (a) The trace of the laser beam transmitted through a transparency between two crossed polarizers and seen on a screen taken with a long exposure photographic picture and (b) three traces of the laser beam (red) transmitted through a transparency between two crossed polarizers and seen on a screen taken with a long exposure photographic picture, while increasing three times the angle $\theta$ from zero to higher values for three different angles $\psi$. The schematic white lines represent areas with constant angles $\psi$, while blue curves represent areas with constant angles $\theta$.

analyser are both rotated until the light is absorbed again. The vibration directions are marked in figure 9(b) below and are not the same as in figure 9(a).

3.2.1. The angle of incidence. As shown by the previous experiment the obliqueness of the incident light drastically changes the properties of the propagating light in an anisotropic material. It influences the vibration directions but also the phase velocity, the propagation direction and the optical length. All that result as variations of polarization states and therefore different transmitted light detected in different position on the conoscopic figure. Experiments 3 and $\mathbf{4}$ show the influences of the incident direction variation on $\theta$ and the azimuth $\psi$ variation of the incident light on the intensity of the transmitted light.

Optical paths of both partial beams differ for two reasons, the wavelengths and the geometrical lengths of both partial beams due to refraction. Strictly speaking the expression for a phase difference $\delta$ has a different form than in equation (5):

$\delta=2 \pi\left(\frac{d^{\prime}}{\left(\lambda_{0} / n^{\prime}\right)}-\frac{d^{\prime \prime}}{\left(\lambda_{0} / n^{\prime \prime}\right)}\right)=\frac{2 \pi}{\lambda_{0}}\left(n^{\prime} d^{\prime}-n^{\prime \prime} d^{\prime \prime}\right)=\frac{2 \pi h}{\lambda_{0}}\left(\frac{n^{\prime}}{\cos \theta^{\prime}}-\frac{n^{\prime \prime}}{\cos \theta^{\prime \prime}}\right)$.

Here $\lambda_{0}$ is the vacuum wavelength, and $n^{\prime} d^{\prime}$ and $n^{\prime \prime} d^{\prime \prime}$ are optical lengths of partial beams having refractive i $n^{\prime}$ and $n^{\prime \prime}$, respectively. A detailed measurement of the polarization state could be performed with a set-up presented in figure 9 following the procedure presented in figure 7. However, to make visually evident the correspondence between the conoscopic figure and the experiment that studies the dependence separately, the laser is tilted instead of the sample (set-up shown in figure 8(c)). The incident angle is controlled by the tilt of the laser with respect to the normal to the anisotropic sample between two crossed polarizers. The intensity of the transmitted light varies with changes of the angle of incidence $\theta$ and the azimuth $\psi$ (figure 10(b)). 


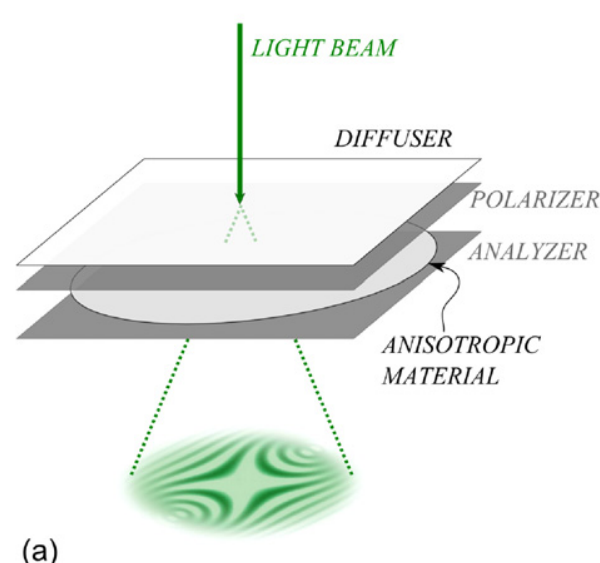

(a)
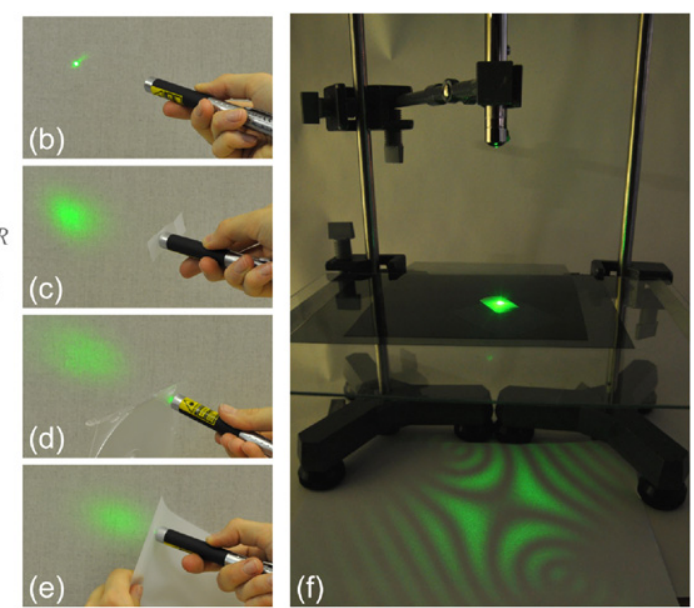

Figure 11. (a) Schematic representation of the experimental set-up, (b) the beam of the laser without diffuser, the laser beam diffused with (c) Scotch tape, (d) translucent CD case and (e) semi-transparent tracing paper and (f) the illumination of a spot on the transparency between two crossed polarizers with a laser covered by Scotch tape and the formation of a conoscopic figure taken with a long exposure photo.

\subsection{The conoscopic figure}

The conoscopic figure presents simultaneously the consequences of all the separate phenomena discussed above. Each position on the figure is associated with a different incident angle and a different azimuth, and consequently, the polarization state of transmitted light in each direction is different due to a different phase difference $\delta$ and due to a different polarization direction of incident light with respect to different vibration directions in the specific direction $\varphi$.

For teaching purposes a simple conoscope (experiment 5) is assembled as follows.

The model conoscope is made by illuminating the sample between two crossed polarizers with a diffused light. The experiment is similar to that described in section 3.2.1, only the sample is illuminated from more angles at the same time. The diffusion of the laser beam can be obtained with translucent (diffuse scattering) Scotch tape (figure 11(c)), a translucent part of some of the microscopic slides, some translucent plastics (from a CD case, figure 11(d)) or other diffusers (as in figure 11(e)). The diffuser should be as close to the sample as possible such that the illuminated area is small and consequently the conoscopic figure is sharp. The polarizers, the sample and the diffuser are put together one on the top of the other. The screen on which the conoscopic figure is formed can be placed at different distances from the sample with respect to the desired size of the figure.

The changes in thickness of the sample and the wavelength of the incident light also influence the polarization state. To avoid such influences one uses monochromatic light and a sample with a uniform thickness. Our goal is to use simple and cheap transparent anisotropic materials, but also those should be chosen carefully, because not all transparent anisotropic materials give a proper conoscopic figure. Several minima and maxima of transmitted intensity of light for different angles $\theta$ (that give several isochromes in the conoscopic figures) are needed. Therefore the sample has to have the appropriate parameters $h, \lambda$ and $\left(n^{\prime \prime}-n^{\prime}\right)$ that define the phase difference and consequently the state of polarization and therefore the transmitted intensity at different incident angles $\theta$ (figure 12). This is the reason that a thin 


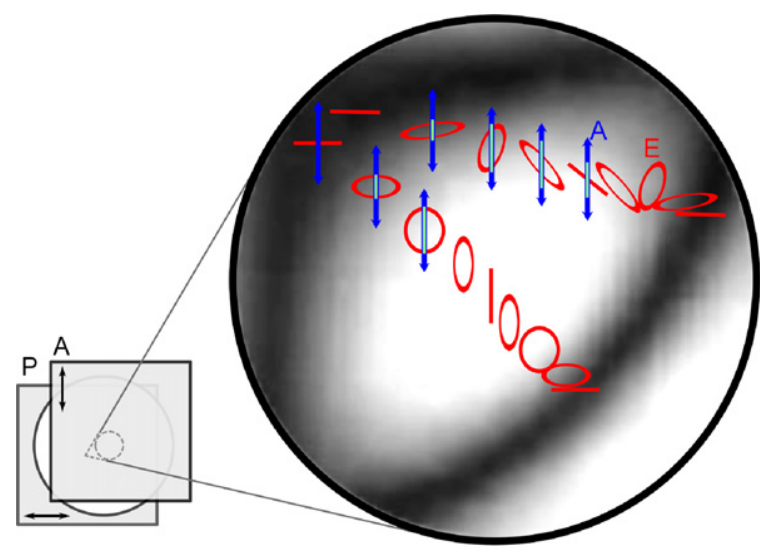

Figure 12. Schematic representation of polarization states (red) of light after passing the polarizer and a sample. The orientation of the analyser is on some polarization states schematically represented with blue double arrows and also the projection of the polarization state to it (light blue), which represent the double amplitude of the transmitted electric field through the analyser. The representation is for a marked part of a uniaxial conoscopic figure with normal axis orientation of the material as seen on the left.
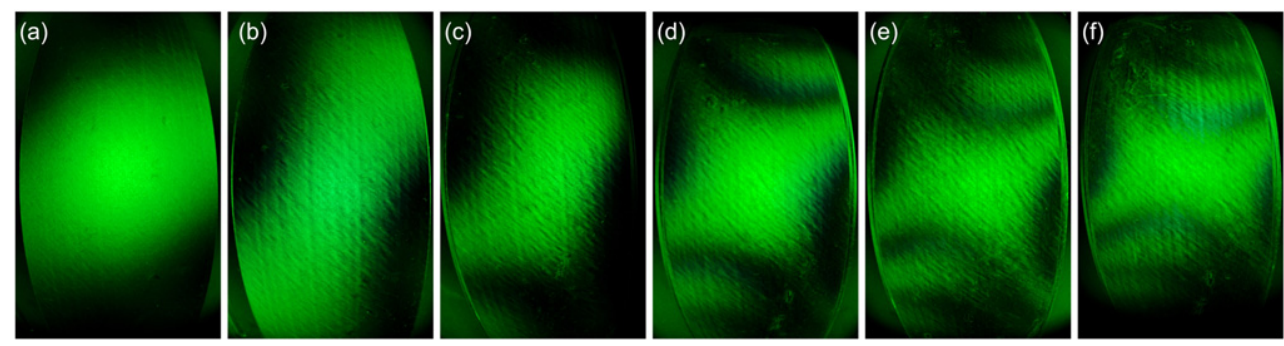

Figure 13. Pictures (taken with a fisheye lens) show the same part of the conoscopic figure for the transmitted monochromatic light through (a) one, (b) two, (c) four, (d) six, (e) eight and (f) ten layers of transparent adhesive tape between two crossed polarizers.

transparency gives a nice conoscopic figure, while a layer of transparent adhesive tape does not. The birefringence $\Delta n$ of transparent adhesive tape is too small. To get a nice conoscopic figure from the transparent adhesive tape one has to increase the thickness as shown in figure 13.

When a hands-on apparatus for observation of conoscopic figures, i.e. a conoscope, is assembled, the lecturer allows students to observe conoscopic figures of different samples from typical anisotropic materials.

The most easily accessible biaxial materials that give a proper conoscopic figure (appropriate thickness and birefringence of the material) are transparencies. A typical conoscopic figure of a biaxial material is seen in figure 14(a). Two dark points, the melatopes, also called bull's eyes, are encircled by bright and dark rings that merge in the middle of the conoscopic figure. The two dark points in the conoscopic figure correspond to the two optical axes determined by directions of light in which the phase velocity is equal for all polarizations. Transparencies usually have the largest $\varepsilon$ in the direction in which the transparent 

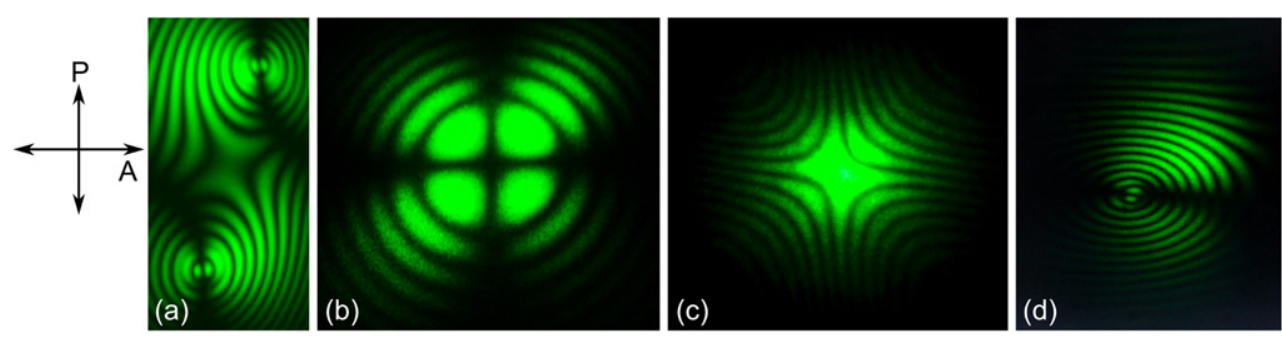

Figure 14. Conoscopic figures of (a) a biaxial sample (transparency, thickness $h=110 \mu \mathrm{m}$ ) (b) a uniaxial material with the axis normal to the sample plane (homeotropically aligned nematic liquid crystal 5CB of thickness $h=200 \mu \mathrm{m}$ ), (c) a uniaxial material with the axis parallel to the sample plane (several layers of adhesive tape with a total thickness of $h=1.2 \mathrm{~mm}$ ) and (d) a biaxial sample (transparency) with random orientation. For all figures the orientation of the polarizer and the analyser is the same (left: schematic representation of $\mathrm{P}$ and $\mathrm{A}$ ).

material was rolled and stretched during the production process. The smallest usually lies in the direction perpendicular to the transparency. If so the two optical axes are in the plane defined by the largest and the smallest epsilon that is perpendicular to the transparency.

A typical conoscopic figure for a uniaxial material is seen in figure 14(b). Here a dark point in the middle corresponds to the single optical axis of the material that is perpendicular to the sample (a typical orientation). The figure is also nicely symmetric with respect to the optical axis. Cheap and easily obtained uniaxial materials having the optical axis perpendicular to the thin sample are not very common, at least to our knowledge. Several crystals are uniaxial, but they should be cut in the specific orientation and to specific thickness to obtain a proper conoscopic figure. Transparent adhesive tapes are also uniaxial [18, 19], but with a planar axial orientation. Materials that are rather easily manipulated are liquid crystals. In the nematic phase of a liquid crystal elongated molecules are parallel. Liquid crystals can be synthesized in a school laboratory [27] or obtained from providers (e.g. Merck [28]). The synthesis described by Pavlin et al takes about 90 min and must be performed in a fume hood. It consists of carefully heating the mixture of 4-butylaniline and p-anisaldehyde to a cloudy liquid, which should be rinsed several times to remove the impurities, as accurately described in [27]. By submerging a microscope slide and cover slip (or two microscope slides) into a solution of simple dishwashing detergent, the surface treatment that provides perpendicular orientation of long molecular axes of the nematic liquid crystal is prepared. A drop of a liquid is put on a dry preliminarily treated microscope slide glass and covered with the cover slip, also treated previously. For accurate thickness of the sample two spacers are used between the glasses. The spacer can be simply adhesive tapes or thin wires strongly fixed to the microscope slide.

The third typical picture is similar for uniaxial and biaxial materials and to distinguish between them additional observation should be made. As in figure 14(c) melatopes are not seen and dark and bright areas form a symmetric picture. Such a situation is met when optical axes are perpendicular to the initial light beam. Materials that provide such a picture are the regular transparent tapes, because they have planar axial orientation due to the stretch in one direction during manufacturing. Nematic liquid crystals provide samples in which the direction of the optical axis can be manipulated. By rubbing clean object and cover glasses with velvet the elongated molecules orient parallel to the glass surfaces along microscopic scratches and the optical axis is correspondingly determined by the rubbing direction [27]. 

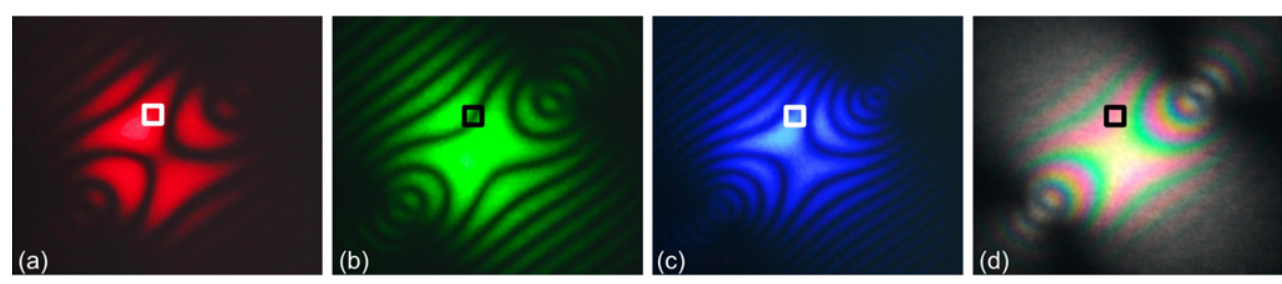

Figure 15. Conoscopic figures of a same point on the transparency illuminated with (a) red, (b) green, (c) blue diffused laser beams and (d) with white light. The square marks the same position on all four conoscopic figures.

A similar conoscopic figure is gained from biaxial materials with axial plane parallel to the surface, but such samples are not so easy to find.

Researchers often observe conoscopic figures of samples having optical axes in general direction, when determining optical properties of anisotropic materials. Experiences of such conoscopic figures are provided by tilting the samples with respect to the laser beam (figure 14(d)).

For a better understanding of conoscopic figure formation or for verification that understanding is correct, we propose some additional experiments.

3.3.1. The wavelength of the incident light and isochromes (experiment 6). In the previous experiments different wavelengths of light were used. The reason was mostly the photographic quality. Does the wavelength of the incident light affect the conoscopic figure and if so, how?

Let us first compare three conoscopic figures from the same material, taken with the same set-up, but with different lasers: red, green and blue (figures 15(a)-(c)). The three figures have the same 'shape' but differ mainly in the 'density of dark lines'. Assuming that the dispersion of refractive indices is negligible, the only parameter that changes for a single position (marked by a square for example) from one figure to another is the phase difference $\delta$ due to the different wavelength. Dark lines correspond to states that are linearly polarized in the same direction as the polarizer after the transmission through the sample and the light is absorbed at the analyser. Therefore the phase difference $\delta$ is equal to a multiple of $2 \pi$. According to the equation (5), the phase difference depends on the wavelength $\lambda$ that crucially defines the optical length. For shorter wavelengths phase differences are larger and vice versa. Therefore in the same direction of incident light and exactly equal refractive indices, the phase difference can result in a dark state at the position that is bright for a different wavelength. For example, at the position marked by a square on figures 15(a)-(c), the phase difference for a red light is close to $5 \pi$ and the position on the red figure will be very bright. For a green wavelength the phase difference is close to $6 \pi$ and the same position on the green figure is dark. For the blue light, the phase difference is close to $9 \pi$ and will be again bright. Observing the same conoscopic figure in the white light (figure 15(d)) one sees that the same position has a magenta colour due to mainly blue and red components.

Here the term isochrome becomes self-explanatory. It corresponds to the curves connecting positions having the same phase difference. The light illuminating positions on one such line in a white light conoscopic figure has a well defined spectrum and consequently the same colour. 

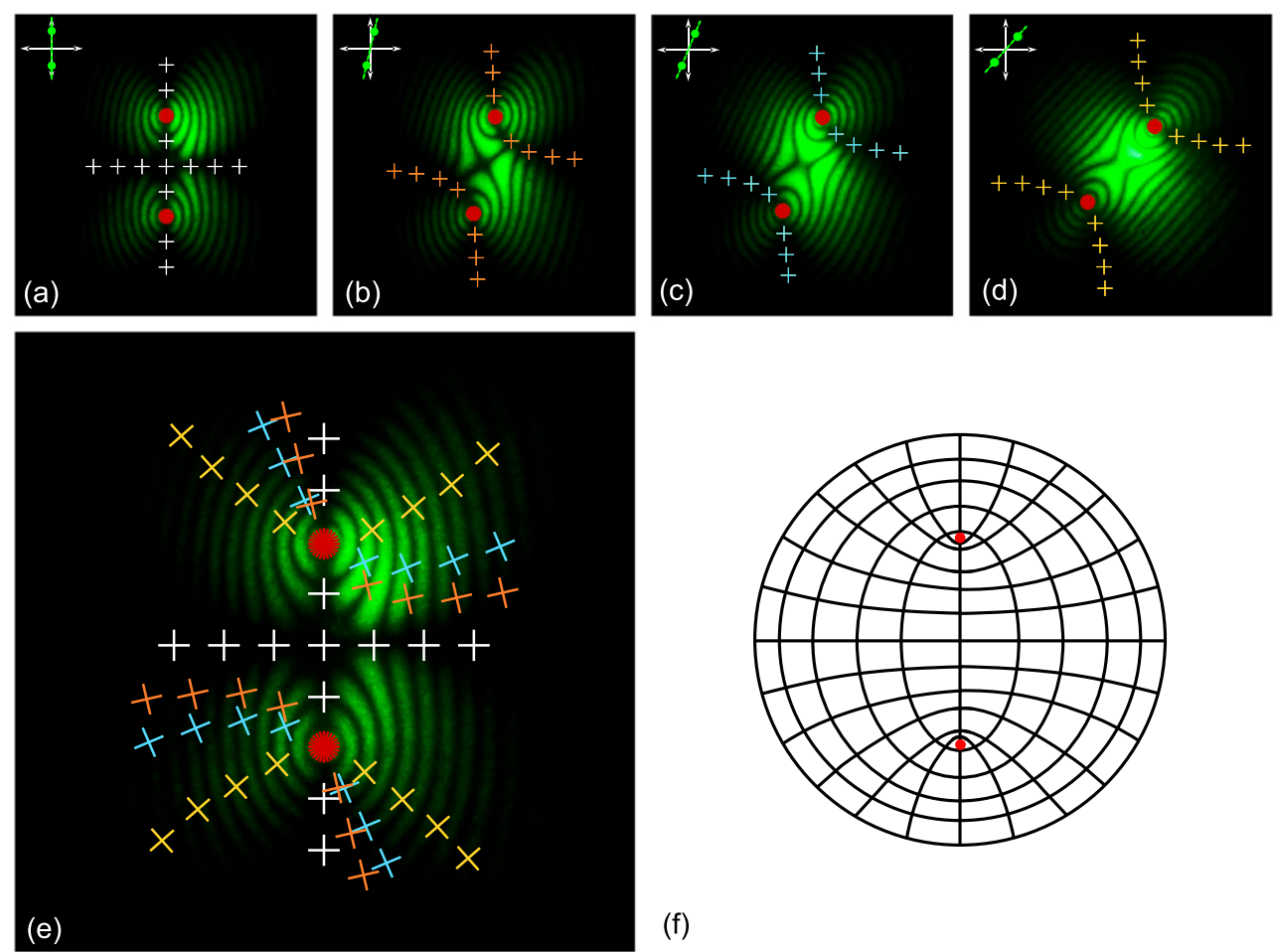

(f)

Figure 16. Conoscopic figures of the biaxial sample (a transparency). The sample is rotated with respect to crossed polarizers that are indicated in the left corner of each picture. Rotating angle is measured between the transmission direction of polarizer $\mathrm{P}$ and the plane containing optical axes seen as melatopes. The sample is rotated for (a) $0^{\circ}$, (b) $13^{\circ}$, (c) $22,5^{\circ}$, and (d) $43^{\circ}$. Vibration directions are marked on the brushesthe dark isogyres. (e) Figure shows isogyres with the angles between the polarization direction and the vibration direction $0^{\circ}$ (white), $13^{\circ}$ (orange), $22.5^{\circ}$ (blue) and $42^{\circ}$ (yellow). This experimental procedure allows for mapping of vibration direction for a general place in the figure. In the directions of the melatopes (optical axes) the marks are red and in those directions the light is travelling as through isotropic material. (f) Skiodrome of a biaxial material.

3.3.2. The brushes and isogyres (experiment 7). Finally, let us observe the changes of the conoscopic figure if the sample is rotated between crossed polarizers.

Figure 16(a) shows a conoscopic figure of a biaxial sample oriented in such a way that two optical axes lie in a plane defined by a transmission direction of the polarizer and the normal to the sample. Both melatopes are encircled by dark isochromes. Two additional dark lines, also called brushes, parallel to transmission directions of both polarizers crossing isochromes are clearly seen. These two lines connect positions in the conoscopic figure that are related to light beams being polarized along one of the vibration directions in the anisotropic sample. White crosses mark these two directions at each point in an upper right part of the figure.

Figure 16(b) shows the same sample rotated clockwise by about $13^{\circ}$ in the same set-up. Brushes now have a different shape. They again cross the melatopes, as the light propagating along the optical axis can have any polarization. Dark brushes that start in the melatope connect positions with the same property as before the vibration directions of both beams correspond to transmission directions of $\mathrm{P}$ and $\mathrm{A}$. Orange crosses in the figure mark vibration 
directions within the upper brush. Rotating further (figures 16(c) and (d)) it is clearly seen by rotating the sample; the brushes have different orientations and forms. This procedure allows for experimental determination of vibration directions for light illuminating each point on the conoscopic figure.

Figure 16(e) shows the cumulative results of vibration direction mapping performed in the first four figures. Crosses indicating different vibration directions were transferred from figures 16(b)-(d) onto figure 16(a) and were rotated to correspond to the system presented in figure 16(a). The colour code indicates crosses from the same figure that correspond to the brushes observed at that figure. It is clearly seen that crosses are rotated for the same angle anti-clockwise as the corresponding figure where they were marked. If orange crosses are connected by a line, the places illuminated by the light with the vibration direction of one of the beams that forms an angle of $13^{\circ}$ with the transmission direction of polarizer in the anisotropic material are known. Such lines with fixed orientation of the polarizer with respect to vibration direction are called isogyres. Different angles are marked by differently coloured crosses on figure 16(e). A greater amount of such mapping would result in a map of curves called a skiodrome that define the planes of vibrations in various directions. For a biaxial material a skiodrome is represented in the figure 16(f). Such experiments not only improve the understanding of isogyres, but also of skiodromes, which are often used for explanation of isogyres and consequently their lines are often mistaken as isogyre curves.

The procedure described above allows for determination of vibration directions of light illuminating any point in the conoscopic figure. It is clearly seen that for oblique incidence the polarization directions change with azimuth of the incident beam as shown already by much simpler experiments (figures 9 and 10). As already said, the conoscopic figure merges several phenomena presented separately by previous introductory experiments.

\section{Conclusions}

The transmission of light through an anisotropic material and its polarization seems to hide a lot of small phenomena and subtle details that contribute to conoscopic figure formation and which are far from clear to many undergraduate students. This paper presents a theoretical background important for understanding conoscopic figure formation and two sets of experiments. The first set of experiments study isolated phenomena that contribute to the conoscopic figure formation. The aim is to provide fundamental experiences to students on how the elliptical state of polarization can be determined, influenced and how it influences the transmission through crossed polarizers. In the second set of experiments, a simple hands-on conoscope is first constructed. This conoscope is then used for experiments introducing elements of the conoscopy: different conoscopic figures for different materials with different properties and different orientations, the influence of the wavelength on the figure, and experimental demonstration of isochromes and isogyres.

We believe that the set of experiments suggested in this paper will help students to construct the necessary knowledge and also stimulate the lecturer to present some of them as a demonstration or to include conoscopy in the optics laboratory if not yet present.

We purposely did not include the extraction of optical data, refractive indices, optical axis orientations and similar from the figure as the procedures are discussed in detail in various textbooks $[22,23]$. Our main aim was to allow students enough experience through experiments to substantiate the extraction of data from experimental measurements for research purposes that are often done by following 'cookbook recipes'. 


\section{Acknowledgments}

The authors gratefully acknowledge Dr Simon Čopar, and the reviewers for their careful reading of the contribution and for useful suggestions.

\section{References}

[1] Zeldovich B Y and Soileau M J 2004 Phys.-Usp. 47 1239-55

[2] Herreman W and Notebaert H 1983 Am. J. Phys. 5191

[3] Morgan B H 1982 Phys. Teach. 20 541-2

[4] Cox A J 1978 Am. J. Phys. 46 302-3

[5] Fernando P C B and Samarasinghe S 1996 Am. J. Phys. 64 565-8

[6] Mayes T W 1976 Am. J. Phys. 44 1101-3

[7] Camp P R 1994 Am. J. Phys. 62 859-60

[8] Perkalskis B S 2010 Am. J. Phys. 781434

[9] Berry M, Bhandari M and Klein S 1999 Eur. J. Phys. 20 1-14

[10] Burns S H and Jeppesen M A 1960 Am. J. Phys. 28 774-6

[11] Davies G R 1990 Phys. Teach. 28 464-7

[12] Ohtsuka K, Ara H and Ogawa T 1984 Jpn J. Appl. Phys. 23 1541-2

[13] Gao Y, Xia H R, Xu J Q, Si S C, Zhang H J, Wang J Y and Song H L 2007 Cryst. Res. Technol. 42 1126-31

[14] Ageev L A and Yegorenkov V D 1999 Am. J. Phys. 67 837-8

[15] Piquero G and Velgas-Balbuena J 2004 Eur. J. Phys. 25 793-800

[16] Bullver-Cebreros C and Rodriguez-Danta M 2009 Eur. J. Phys. $30285-94$

[17] Ziherl S, Bajc J, Urankar B and Čepič M 2010 Eur. J. Phys. 31 531-42

[18] Blanco M A, Yuste M and Carreras C 1997 Am. J. Phys. 65 784-7

[19] Beléndez A, Fernández E, Francés J and Neipp C 2010 Eur. J. Phys. 31 551-61

[20] Cloud S D 1973 Am. J. Phys. 41 1184-8

[21] Trester S 1993 Am. J. Phys. 61 62-6

[22] Born M and Wolf E 1993 Principles of Optics (Oxford: Pergamon)

[23] Hecht E 2002 Optics 4th edn (San Francisco: Addison-Wesley)

[24] EMANIM 2014 Animations of electromagnetic waves www.enzim.hu/szia/emanim/emanim.htm (24 February 2014)

[25] Hartshorne N H and Stuart A 1964 Practical Optical Crystallography (London: Edward Arnold)

[26] Wahlstrom E E 1969 Optical Crystallography 4th edn (New York: Wiley)

[27] Pavlin J, Susman K, Ziherl S, Vaupotič N and Čepič M 2011 Mol. Cryst. Liq. Cryst. 547 255-61

[28] Merck \& Co. Inc., www.merck.com/ 